\title{
Malignant Gastrointestinal Stromal Tumor
}

\author{
P ISSAR, MK DWIVEDI, SK ISSAR, RK PAL, L DEWANAGAN
}

Ind J Radiol Imag 2006 16:1:65-67

\author{
Key words : - GIST USG, CT \\ Introduction
}

Gastrointestinal stromal tumors formely classified as leiomyomas or leiomyosarcomas are mesenchymal tumors of the gastrointestinal tract that differ from true Leiomyomas and Leiomyosarcomas. Classification of mesenchymal tumors of the gastrointestinal tract has been the subject of controversies for many years and several histological classification system has been proposed. GIST are now defined as spindle cell, epitheloid or occasionally pleomorphic mesenchymal tumors of the gastrointestinal tract without smooth muscle cell or schwann cell differentiation $(1,3)$. The term GIST is also limited to tumors originating from pacemaker cell of Cajal located between myenteric plexus cells and smooth muscle cells of the GIT. The immuno-histo chemical marker 'C-Kit' (CD-117) identifies these cell and seems to be the most specific diagnostic marker currently available $(1,3)$. We present two cases of GIST with their imaging and histological features.

\section{CASE I}

A 19 year old man was admitted with the complain of abdominal pain weakness and hematemesis. On laboratory examination his hemoglobin was $4.2 \mathrm{gm} / \mathrm{dl}$ (macro cystic hypo chromic anemia). Hemoglobin electrophoresis did not reveal any abnormal hemoglobin. On ultrasound examination a heterogenous mass lesion was seen in lesser sac in relation to posterior wall, body of stomach, stomach was pushed up and to the right. Pancreas was compressed by the mass lesion but showed normal echo texture (Fig 1). Pre and Post contrast CT was obtained and showed a large mass $(14.2 \times 10.4$ $x 9.1 \mathrm{~cm}$ ) along the posterior wall of the greater curvature of the stomach. The mass was well defined, heterogenous with multiple cystic foci and solid component showing enhancement equivalent to Liver (Fig 2). On the basis of ultrasound and CT a diagnosis of exophytic Leiomyoma was made.

Gastroscopy showed a large sub mucosal tumor just below the gastro - oesophageal junction upto antrum over posterior wall. In the body region it was ulcerated and nodular with evidence of active bleeding. Endoscopic biopsy was obtained that was consistent with GIST. Complete gastrectomy was performed with out complication. On Gross examination a large encapsulated sub serosal mass with a cystic component containing blood and solid whitish component with hemorrhagic foci seen. On histopathological examination diagnosis of GIST was confirmed.

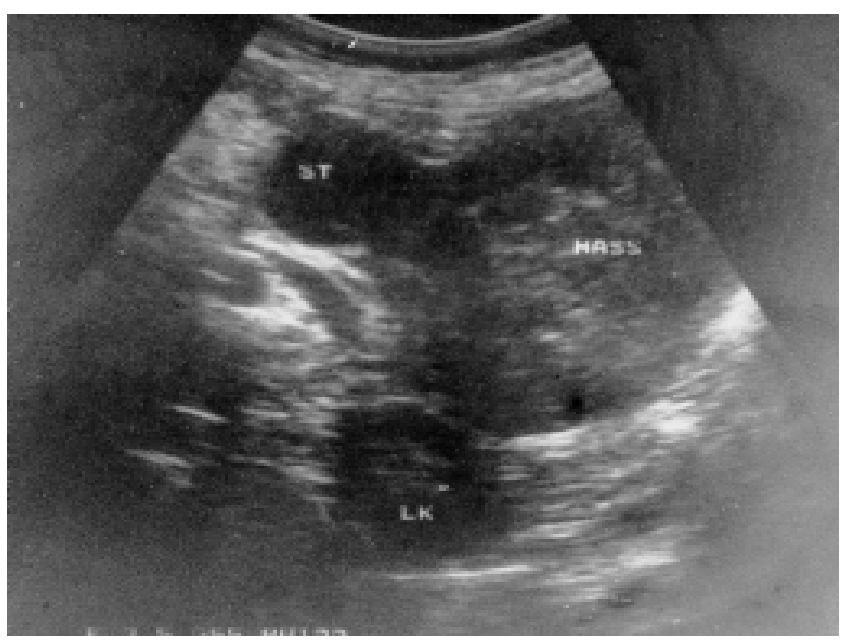

Fig 1

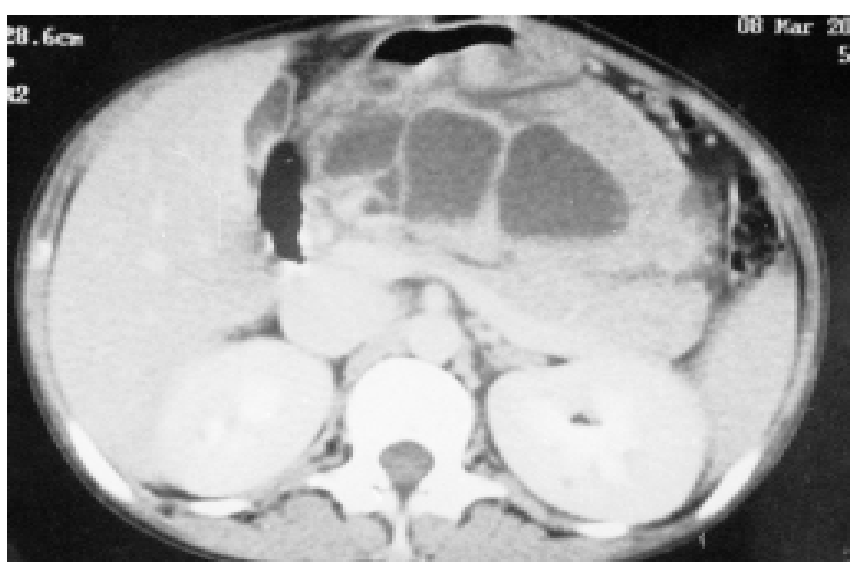

Fig 2

From the Dept. of Radiodiagnosis, JLN hospital \& Research Centre, Bhilai

Request for Reprints: Dr. Pratibha Issar, Mohini Kunj, Saket Colony, P.O. S A F Lines, Katul Board Durg Bhilai (C.G.) 490022 
66 P Issar et al

\section{CASE II}

A 63 year old woman was admitted with a history of reduced appetite since one month along with pain and low grade fever of 5 days duration On examination a palpable non tender mass was present in mid abdomen region.

U.S. showed a large heterogenous mass lesion in lesser sac region, occupying epigastrium and Lt. hypochondrial region with outer solid and centre cystic area. Stomach was pushed up and to the right and left kidney was pushed down. Normal pancreas was seen in the head region. Liver was normal (Fig 3).

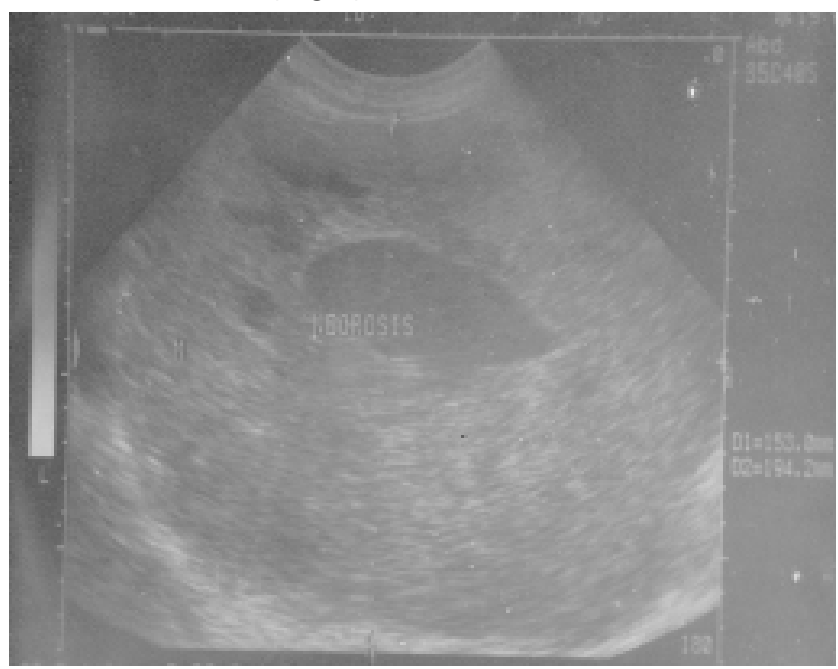

Fig 3

On CT examination a heterogenous mass lesion measuring $20.4 \times 12.8 \times 23.1 \mathrm{~cm}$ seen in relation to posterior wall of fundal region of stomach and pushing it anteriorly towards Rt. Duodenum second part was also pushed towards Rt. Lt. kidney was pushed down. Mass was extending upto splenic hilum (Fig 4). On the basis of U.S. and CT a possibility of exophytic gastric Leiomyoma or Leiomyosarcoma was made.

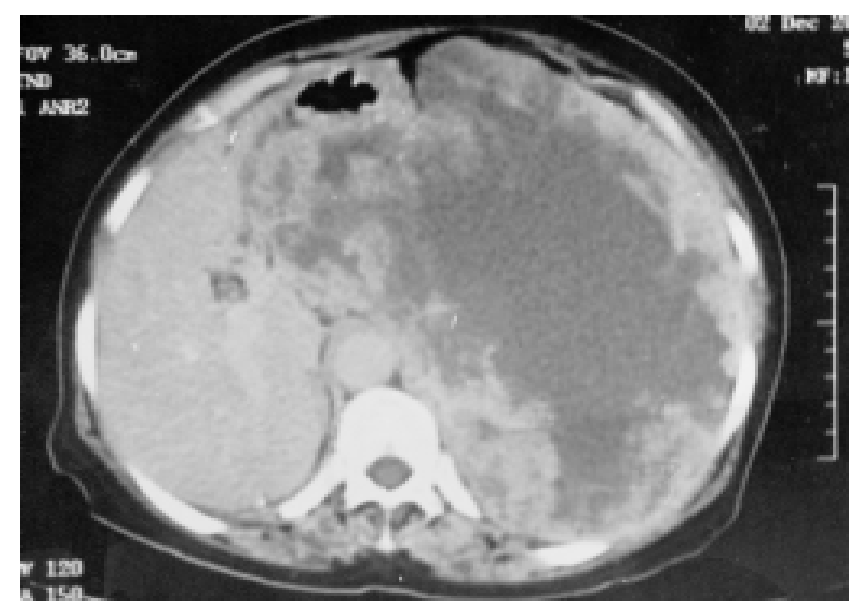

Fig 4
IJRI, 16:1, February 2006

FNAC of the lesion showed highly cellular smears with presence of fascicles of spindle shaped cells in a suspended background along with few mitosis and atypical nuclei. On the basis of this a diagnosis of GIST was made.

On surgery a large mass was seen occupying lesser sac, displacing stomach up and towards Rt. and transverse colon down and extending upto splenic hilum. It was seen arising from fundus of stomach. Removal of mass along with partial gastrectomy with splenectomy was done. Histopathology confirmed it as malignant GIST.

\section{Discussion: -}

GIST are rare but are nevertheless the most common mesenchymal neoplasms of the Gastro-intestinal tract. GIST are most frequently found in stomach (60-70 \%) followed by the small intestine $(20-30 \%)$, the colorectum $(10 \%)$ and the oesophagus $(65 \%)(4,5)$

They account for $2-3 \%$ of all gastric tumors. They may also occur as primary tumors out side the gastro intestinal tract, especially in the omentum mesentery and retro peritoneum. The term stromal tumor has several accepted definitions in the literature. A general definition of mesenchymal tumors includes all mesenchymal tumors originating from spindle cells, (irrespective of their differentiation into leiomyoma, leiomyosarcoma, schwannoma, tumors of autonomic nervous system) A more restrictive definition of GIST would include only tumors containing spindle or epithelioid cells without smooth muscle cell or schwann cell differentiation. Recently the name gastro intestinal pacemaker cell tumor is used since most of these tumours would originate from pacemaker cells of Cajal. Pacemaker cells are identified by the immuno histochemical marker C-Kit and are located between the myenteric plexus and somooth muscle cells of the GI wall, However presence of the receptor in omental tumors raises questions regarding the exclusive origin of stromal tumors from pacemaker cells. (I, 4,5,6)

The most common clinical manifestation of symptomatic stromal tumor in the GIT is gastrointestinal bleeding caused by mucosal ulceration. Patients may present with hematemesis, melena, hematochezia or signs and symptoms of anemia caused by occult bleeding. Other symptoms are abdominal pain and a palpable mass or patient may remain asymptomatic $(2,7)$.

A palpable mass may be present when the tumor is larger and predominantly exophytic. Bowel obstruction is quite uncommon and patient can be febrile which could be an indicator for malignant degeneration. In patients with a large intra abdominal mass determination of the site or origin of the tumor can be difficult and multimodality 
IJRI, 16:1, February 2006

imaging often is required to further characterize the site of origin of such masses $(2,3)$

Upper GI Barium studies are abnormal in about $65 \%$ of cases and this examination is mainly useful to detect tumor with endoluminal component since exopytic component con not be differentiated from extrinsic compression and mass effect.

On US GIST are seen as well defined mass lesion of varying sizes and echogenicity often with central cystic areas. Their gut origin is not always easily determined but if ulceration is present, pockets of gas in an ulcer crator may suggest a tumor with gut origin.Endoscopic US is useful to confirm the gastric origin of the mass but cannot confirm or exclude the presence of malignant degeneration.(1)

Upper $\mathrm{Gl}$ endoscopy is abnormal in about $71 \%$ of cases, but cannot always confirm the gastric origin of the mass. If the GIST is mainly exophytic , endoscopy may be normal or only shows nonspecific secondary findings (Ulceration of the overlying mucosa) (1)

The diagnosis of malignant GIST can be suggested on CT scans with the presence of a large well-circumscribed tumor arising from the stomach or small bowel that is usually predominantly extraluminal and has a heterogeneously enhancing soft tissue rim surrounding a necrotic center. Metastases if present will be to liver or peritoneum. Lymph node enlargement is not a feature. Differential diagnosis are Lymphoma, Carcinoids, Metastases to the bowel, Leiomyosarcoma, Malignant tumor of nerve sheath etc.
Malignant Gastrointestinal Stromal Tumor 67

In conclusion the radiologic appearance along with the pathological finding is helpful in diagnosis of malignant GIST.

\section{References: -}

1. F Mignon, C Julie, R. Izzillo etal. Imaging Features of Gastric Stromal Tumores : Radiologic Pathologic correlation. Review of Four cases. Journal de radiologic 2000; 81: 874-881.

2. Toshirore Nishida, Seishi Kumano, Takashi Sugiura etal. Multidetector CT of High - Risk Patients with Occult Gastrointestinal Stromal Tumors AJR January 2003; 180: 185-189.

3. Gny J.C Burkill, Mohammed Badran, Omar Al - Muderis etal. Malignant Gastrointestinal Stromal Tumor: Distribution Imaging Feature, and Pattern of metastatic Spread. Radiology 2003; 226: 527-532.

4. Hyo-Cheolkim, Jeong Minlees, Se Hyungkim etal. Primary Gastrointestinal Stromal Tumors in the Omentum and mesentery : CT Findings and pathologic Correlation. AJR June 2004; 182: 1463-1467.

5. Hyo-Cheol Kim, Jeong Minlee, Kyu Rison etal. Gastrointestinal Stromal Tumors of the Dueodenum : Ct and Barium Study Findings. AJR August 2004; 183: 415419.

6. Rosai and Ackerman's Surgical Pathology Ninth Edition Vol. Stomach (Stromal Tumors GIST and Releated lesions). Mosby Publication 2004.. Page 674-680

7. Hyo- Cheol Kim, Jeong MinLee, Kyoung Wlion Kim at all. AJR Gastrointestinal Stromal Tumors of the stomach: CT Findings and Prediction of Malignancy. AJR October 2004; 183: 893-898. 


\section{Announcement}

The Department of Radiodiagnosis, SGPGIMS, Lucknow is organizing a three day CME program on "CLINICALAPPLICATIONS OF NEWER MRI TECHNIQUES IN NEUROSCIENCES" from 9th February 2007 to 11th February 2007. The basic concepts, technique, analysis, and clinical applications will be dicussed in detail along with practical clinical demonstration on MR scanner. The aim is to have a limited number of delegates for a meaningful and interactive discussion. Faculty for this CME comprises of eminent International and National experts workingin the field of MR. The registration fee of Rs. 2000/- will include boarding and lodging at the Guest House at SGPGIMS. The weather in early February will be pleasant albeit a bit on the cooler side.

Interested persons should contact the following for registration.

Prof Rakesh K Gupta

rgupta@sgpgi.ac.in

tel 05222668700 extn 2599
Prof Sunil Kumar

sunilk@sgpgi.ac.in

05222668700 extn 2574

Department of Radiodiagnosis SGPGIMS

Rae Bareli Road,

Lucknow-226 014 